\title{
Tilted Bragg Gratings as an Efficient Platform for Integrated Multimode Interference Devices
}

\author{
Peter Horak*, Mathias J. Weisen, James C. Gates, Corin B. E. Gawith, Peter G. R. Smith \\ Optoelectronics Research Centre, University of Southampton, Southampton SO17 1BJ, United Kingdom \\ *e-mail:peh@orc.soton.ac.uk
}

\begin{abstract}
We have recently developed and experimentally demonstrated the technology of tilted Bragg gratings in integrated optical waveguides to achieve polarisation selective coupling between parallel waveguides. Here I will review our ideas, designs and simulations for novel devices based on this platform. Multimode devices, tailored phase matching, and methods for significantly improved coupling efficiencies will be discussed that will be of interest for applications in quantum information processing and multimode optical telecommunication.
\end{abstract}

Keywords: Integrated photonics; Bragg gratings; phase matching; multimode waveguides; mode coupling.

Direct UV writing is a laser manufacturing technique for integrated optical waveguides and Bragg gratings in hydrogenated germanium-doped silica-on-silicon chips. This method allows us to write tightly controlled nanostructures, and in particular to write tilted Bragg gratings where the grating planes are at arbitrary angles with the waveguide. As one sample application of this technology, we recently demonstrated an integrated polariser. Here the gratings are tilted at the Brewster angle of $45^{\circ}$ degree, which results in near perfect separation of the waveguide TE and TM modes. Experimentally we observed a polarisation extinction ratio of $28.5 \mathrm{~dB}$ [1]. Numerically, we have also shown that the method can be extended to multiport beam splitting and switching in arrays of planar waveguides [2].

The efficiency of such devices can be greatly improved if the waveguide modes are made to interact with a tailored cladding that only supports a few discrete modes, rather than a continuum of radiation modes as in our earlier work. Such a geometry can be realised, for example, by two direct UV written waveguides sitting on a ridge fabricated by micromachining. Ideally, Bragg gratings can then be tuned such that the two waveguide modes are coupled exclusively to a single discrete cladding mode. A grating tilt angle is again required to phase match the waveguide and cladding modes not only in the propagation direction but also in the transverse direction, similar to blazed diffraction gratings. Simulations based on coupled mode theory and full finite element analysis show that, in principle, up to $100 \%$ efficient waveguide coupling can be achieved [3]. By exploiting backward propagating cladding modes, the operation bandwidth becomes extremely narrow (subnanometre in wavelength) and thus the device can act as a narrow-band add-drop filter. In addition, a phasesensitive switch can be realised when light is launched simultaneously into both waveguides: depending on the relative phase $\phi$ of the two fields, light is either completely reflected $(\phi=0)$ or completely transmitted via a grating induced transparency effect $(\phi=\pi)$.

Further degrees of freedom can be exploited if the UV written waveguides are made to support two or more spatial modes by increasing the refractive index contrast or increasing the waveguide width. In this case, a large number $N$ of input channels (different modes in different waveguides) can be supported in a compact device. Grating-induced coupling between waveguide and cladding modes can be made highly selective, and arbitrary $N$-by- $N$ coupling matrices can be achieved [4].

We envisage that devices based on such designs will find applications in classical and, in particular, in quantum information processing. Narrow band filtering, mode-division multiplexing, single-photon or multiphoton interference, optical gates, phase-selective switches and other functionalities can be realised in this compact and highly robust platform.

\section{ACKNOWLEDGEMENTS}

This work was supported by the UK National Quantum Technology Programme under EPSRC grants EP/M013243/1 and EP/M013294/1.

\section{REFERENCES}

[1] M. T. Posner et al.: Integrated polarizer based on $45^{\circ}$ tilted gratings, Opt. Express, vol. 27. pp. 1117411181, Apr. 2019.

[2] N. Podoliak et al.: Optical beam splitting and switching based on arrays of tilted Bragg gratings in planar waveguides, in CLEO-Pacific Rim 2017, Singapore, Aug. 2017, paper P3-075.

[3] M. Weisen et al.: Planar waveguide coupler based on tilted Bragg gratings and a discrete cladding mode, in SPIE Photonics Europe, Strasbourg, France, Apr. 2018, paper 10672-135.

[4] M. J. Weisen et al.: Four-port interference device on an integrated photonics platform based on tilted Bragg gratings, in CLEO/Europe-EQEC 2019, Munich, Germany, June 2019, paper EB-P.7. 